\title{
Modulation of Acridinium Organophotoredox Catalysts Guided by Pho- tophysical Studies
}

\author{
Christian Fischer, ${ }^{\ddagger}$ Christoph Kerzig, ${ }^{\ddagger}$ Bouthayna Zilate, Oliver S. Wenger* and Christof Sparr* \\ Department of Chemistry, University of Basel, Basel CH-4056, Switzerland
}

\begin{abstract}
Control over redox states and spin multiplicity of photocatalysts throughout a catalytic cycle is crucial for selective and efficient photocatalytic processes. However, the rational design of photocatalysts is often hampered by the mechanistic complexity and low modularity of catalyst structure. Herein, we demonstrate a photophysical study of diverging photocatalytic pathways that guides the design of organic acridinium catalysts to complement polypyridyl transition metal systems. A combined halogen-metal exchange / directed ortho-metalation provides reagents for a broad range of modular acridinium catalysts with fine-tuned photophysical and photochemical properties such as excited-state lifetimes, redox potentials and photostabilities poised to refine organocatalytic photoredox methodology.
\end{abstract}

KEYWORDS: acridinium salts, catalyst design, energy transfer, metalation, photoredox catalysis

\section{INTRODUCTION}

Synthetic methodology via radical intermediates has witnessed a dramatic advance by the advent of preparative visible light photoredox catalysis. ${ }^{1}$ A broad range of radical disconnections and mild reaction conditions allowed progressive retrosynthetic planning, expeditiously embraced by academic and industrial laboratories. The resulting versatility of visible light photoredox catalysis is reflected by numerous new reaction types developed within the last ten years. A majority of these methods rely on polypyridyl transition metal complexes and in particular ruthenium and iridium catalysts. ${ }^{2}$ While polypyridyl systems can be readily modulated by changes in the ligand structure, organic photocatalysts, ${ }^{3}$ which render photoredox methods sustainable and more amenable to scale up, remain limited in their tunability. However, compared to polypyridyl ligand modulations, molecular scaffold variation of organic photoredox catalysts requires the development of more general synthetic methods and therefore, most transition metal complex catalyzed reactions are yet not complemented by organocatalytic methods.

For instance, the acridinium organophotoredox catalysts introduced by Fukuzumi ${ }^{4}$ and further developed by Nicewicz ${ }^{5}$ are now established as particularly valuable catalytic motifs for preparative organic synthesis, but their traditional assembly severely limits synthetic modularity. Recently, we developed a method to access acridinium salts directly from carboxylic acid esters ${ }^{6}$ and observed the interesting photoredox activity of diaminoacridinium salt $\mathbf{1}$ in a Ni-dual catalytic cross-coupling reaction (Scheme 1, top pathway). ${ }^{7,6 a}$ This finding and the captivating prospect of a modular organophotocatalyst synthesis prompted us to devise a detailed understanding of the photophysical characteristics of $\mathbf{1}$, guiding a versatile heterocycle synthesis towards broadly applicable acridinium catalysts. A comparative study of the novel acridinium catalysts together with prototypical organic photocatalysts ${ }^{3,8}$ furthermore underlines the significance of complementarity in photoredox catalysis.

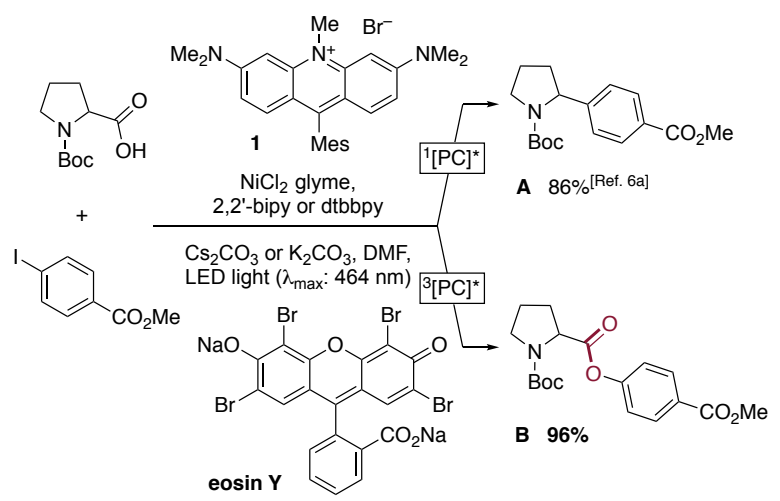

Scheme 1. Divergent photochemical pathways

\section{RESULTS AND DISCUSSION}

We initiated our studies with the dual catalytic decarboxylative cross coupling and an array of most commonly employed organocatalysts was compared to the acridinium system $\mathbf{1}$. Notably, selectivity for the photoredox process to give $\mathbf{A}$ was observed exclusively for diaminoacridinium catalyst $\mathbf{1}$. With a large number of other routinely used organic photocatalysts such as eosin Y, a diverging photochemical triplet-triplet energy transfer (TTET) pathway to sensitize nickel(II) species $^{9}$ provided $O$-aryl ester B with up to $96 \%$ yield (Scheme 1, bottom pathway). ${ }^{10}$ Furthermore, with established highly oxidizing acridinium catalysts such as mesityl-2,7-dimethyl-10phenylacridinium $^{5}$ or the Fukuzumi catalysts, either a mixture of photoredox- and TTET products (A 10\% vs. B 16\%) or no product formation at all were observed. Given the unique reactivity of the diaminoacridinium system catalyzing the selective formation of decarboxylated A, comparative DFT computations of $\mathbf{1}$ and the Fukuzumi catalyst were performed, revealing the fundamentally different nature of lowest energetic electronic transitions in these two compounds (Figure 1). The in- 
troduction of the dimethyl amino groups evidently causes a change from charge transfer $(\mathrm{CT})^{4 \mathrm{~b}}$ to $\pi-\pi^{*}$ character. ${ }^{10}$ The CT states of the Fukuzumi and related catalysts are highly reactive and even able to oxidize anthracenes and stilbenes to their radical cations. ${ }^{4 b, 4 c, 5}$ In contrast to those highly oxidizing properties often resulting in compromised yields, ${ }^{10}$ excited $\mathbf{1}$ with its less energetic $\pi-\pi^{*}$ states is well suited for dual catalysis with nickel and compatible with the components of a complex reaction mixture.

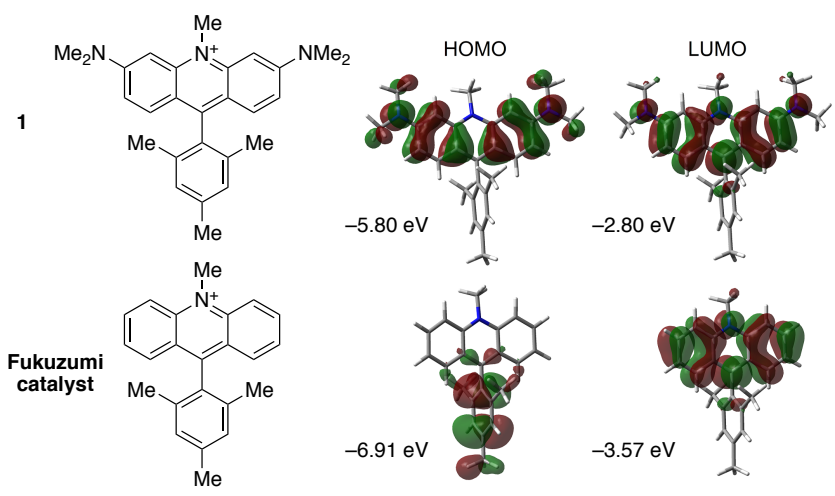

Figure 1. DFT calculations (B3LYP/6-31+G(d,p), IEFPCM solvation with $\mathrm{MeCN}$ ) of $\mathbf{1}$ and the Fukuzumi catalyst. See SI for details.

Interestingly, a triplet energy (ET) for 1 of $1.89 \mathrm{eV}$ was estimated by computation, which is identical to the triplet energy of eosin Y $(1.89 \mathrm{eV}){ }^{3 \mathrm{e}}$ Since the triplet sensitization of aryl nickel carboxylates requires an ET of $>1.85 \mathrm{eV},{ }^{9 a}$ both photocatalysts would be thermodynamically capable of promoting ester formation via their triplet excited states. On the other hand, only the singlet states of both catalysts (ES: $2.40 \mathrm{eV} / 2.30 \mathrm{eV}){ }^{1 \mathrm{f}, 6 \mathrm{a}}$ which can both provide an excited-state reduction potential of about $+1.25 \mathrm{~V}$ vs. SCE, ${ }^{1 \mathrm{f}, 3 e, 6 \mathrm{a}}$ would allow the oxidation of the carboxylate. ${ }^{11} \mathrm{We}$ therefore envisaged that these comparable driving forces, for both triplet sensitization of the intermediate nickel complex and excited singlet state quenching by electron transfer from carboxylates, provide a suitable platform to differentiate the relative contributions and kinetics of singlet and triplet excited states.

Employing laser flash photolysis (LFP) studies, we first observed a long-lived ( $\tau: 330 \mu \mathrm{s})$ species after green laser excitation of 1 (Figure 2); its TTET reactivity towards aromatic hydrocarbons such as anthracene (as opposed to the Fukuzumi catalyst, which undergoes electron transfer) allowed us to identify that species as the triplet state of $\mathbf{1}$ (see SI page S5 for details). Quantitative LFP experiments ${ }^{12}$ on the sensitized ${ }^{3}$ anthracene formation with ${ }^{3} \mathbf{1}$ as energy donor gave an ISC (triplet) quantum yield for $\mathbf{1}$ of about $7 \%$. A kinetic analysis of our TTET experiments finally yielded the experimental triplet energy of the acridinium dye $\mathbf{1}(1.91 \mathrm{eV})$, which is very close to the calculated value (see above). Compared to eosin Y (triplet quantum yield of $80 \%)^{13}$ whose triplet is usually considered as photoactive species, ${ }^{3 e}$ the importance of the excited singlet state of acridinium photoredox catalysts can be further substantiated in agreement with the studies by Nicewicz as discussed below. ${ }^{14}$ Nevertheless, in a photochemical transformation devoid of competitive reaction pathways, the triplet photochemistry of $\mathbf{1}$ remains operative for a classical stilbene photoisomerisation $^{15 a}$ giving the cis isomer in $71 \%$ yield. This conclusion is borne out by LFP studies on the TTET reaction between $\mathbf{3}^{1}$ and pyrene, ${ }^{10}$ which has almost the same triplet energy as trans-stilbene, and the fact that singlet excited 1 cannot oxidize stilbene for thermodynamic reasons. ${ }^{15 \mathrm{~b}}$

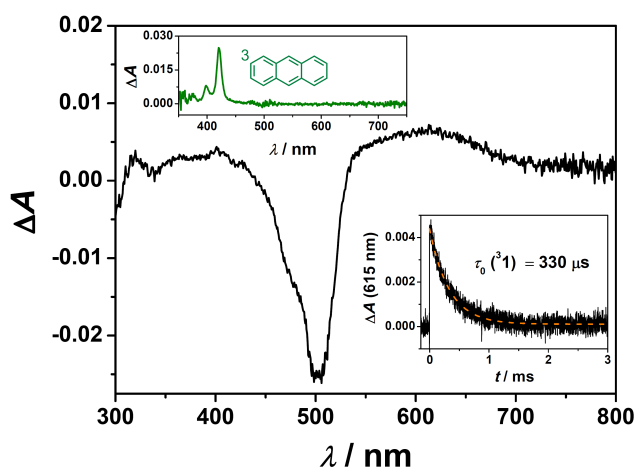

Figure 2. Nanosecond laser flash photolysis (LFP) investigations of the triplet state of $1(20 \mu \mathrm{M}$ in deoxygenated $\mathrm{MeCN})$ using $532 \mathrm{~nm}$ laser pulses of $\sim 10 \mathrm{~ns}$ duration (pulse energy, $16 \mathrm{~mJ}$ ). Main plot, transient absorption spectrum $500 \mathrm{~ns}$ after the laser pulse and time-integrated over $200 \mathrm{~ns}$. Lower inset, kinetic transient absorption trace monitored at $615 \mathrm{~nm}$. Upper inset, transient absorption spectrum in the presence of anthracene $(0.4 \mathrm{mM})$ recorded with a time delay of $3 \mu \mathrm{s}$. See SI for details.

Fluorescence experiments to probe the excited singlet reactivities of catalyst 1 and eosin Y were carried out with Bocproline derived Cs- and K-carboxylates (Figure 3). Despite identical thermodynamics for carboxylate oxidation and a much longer natural lifetime of singlet-excited eosin Y, efficient quenching occurs only with excited 1 (in accord with the observed C-C coupling product A). The influence of Coulomb interactions on the electron transfer kinetics may provide a rational explanation for the notably different rate constants of excited singlet state quenching by anionic substrates (see SI page S6 for details).

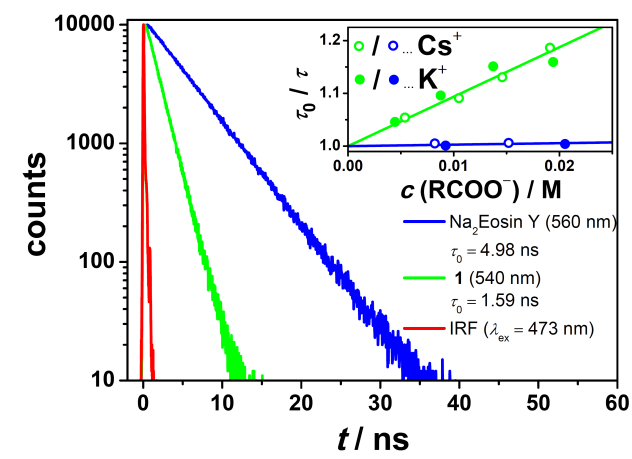

Figure 3. Fluorescence quenching studies of $\mathbf{1}$ and eosin $\mathrm{Y}$ in deoxygenated DMF upon $473 \mathrm{~nm}$ excitation. Main plot, response function of the TCSPC instrument (IRF) and unquenched lifetimes of both emitters. Inset, Stern-Volmer plots using either $\mathrm{K}^{+}$or $\mathrm{Cs}^{+}$-salt of Boc-L-proline as quencher.

Based on these photochemical considerations, we conclude that in comparison to eosin $\mathrm{Y}$, both the lower triplet quantum yield of $\mathbf{1}$ and the higher kinetic reactivity of its excited singlet state for carboxylate oxidation give rise to the divergent pho- 
tocatalytic reaction pathways illustrated in Scheme 1. Guided by the notable photophysical differences between the aminoacridinium system 1 and the established Fukuzumi and Nicewicz catalysts, ${ }^{5}$ we set out to explore the generality of these characteristics with an entirely modular acridinium core structure assembly, providing a wide range of redox properties akin to Ir- and Ru-catalysts. In contrast to our double X-M exchange ${ }^{6 a}$ reactions to 3,6-diamino- and the double directed orthometalation methods $(\mathrm{dDoM})$ giving 1,8-dimethoxy-acridinium salts, ${ }^{6 b, c}$ we investigated an integrated synthesis of amino- and methoxy-acridinium salts by combining X-M exchange and DoM processes (Table 1).

Table 1: Modular synthesis of amino- and methoxyacridinium salts ${ }^{a}$

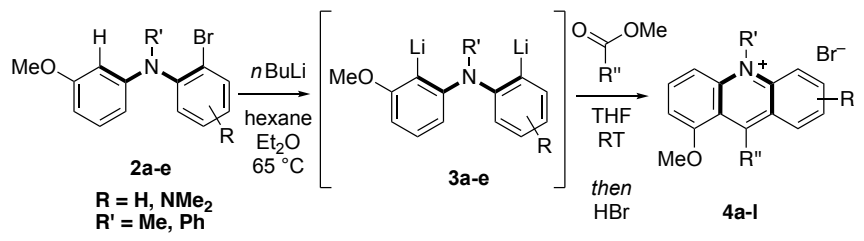

$\begin{array}{lll}\text { Product }^{b} & \text { Product } & \\ & & \text { Product } \\ \end{array}$
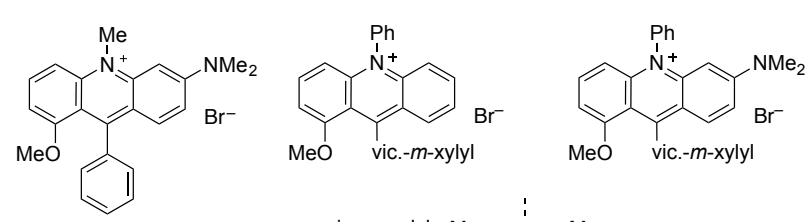

4a, $98 \%$

$4 \mathrm{e}, 71 \%$

$4 \mathbf{i}, 78 \%$
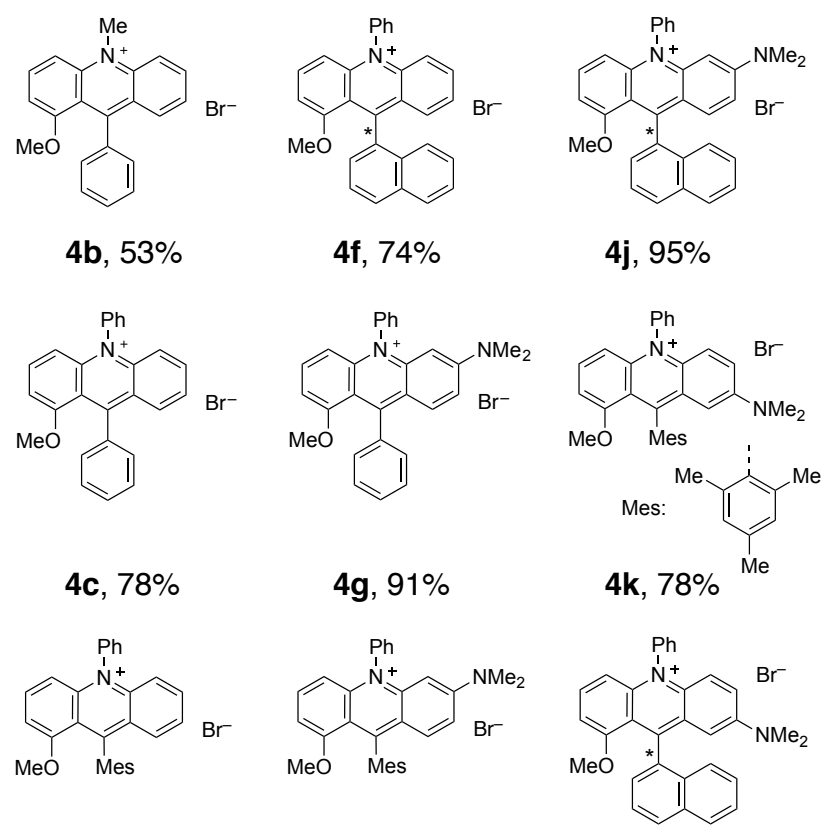

4d, $71 \%$

4h, $76 \%$

4I, $94 \%$

${ }^{a}$ The combined DoM/Br-Li exchange was carried out with 2a-e $(160 \mu \mathrm{mol})$ in a mixture of $\mathrm{Et}_{2} \mathrm{O}(200 \mu \mathrm{L})$ and $\mathrm{n}$-hexane $(2.0 \mathrm{~mL})$ with $\mathrm{n}$-BuLi at $65^{\circ} \mathrm{C}$ for $6 \mathrm{~h}$. Ester substrates $(100 \mu \mathrm{mol})$ were treated with resulting reagents $3 \mathrm{a}-\mathrm{e}$ at a temperature of $-20^{\circ} \mathrm{C}$ and for $12 \mathrm{~h}$ at RT followed by aqueous work-up with $\mathrm{HBr}$. ${ }^{b}$ Isolated yields.
Di- or triaryl amines 2a-e bearing a directing group and bromide with or without amino substituents at distinct positions are readily accessible, rendering this method particularly versatile. ${ }^{10}$ Gratifyingly, the combined X-M exchange/DoM double metalation with $\mathrm{nBuLi}$ at $65^{\circ} \mathrm{C}$ afforded 1,5-dilithium organyls 3a-e, that convert various esters into a broad range of unsymmetric acridinium salts $\mathbf{4 a - 1}$.

To our delight, the reagent from the combined X-M exchange/DoM double metalation gave the phenyl acridinium product $4 \mathbf{a}$ in an excellent yield of $98 \%$ and also the sterically demanding mesityl and vic.-m-xylyl substituted products were reliably obtained $(\mathbf{4 e}, \mathbf{4 i})$. Interestingly, both the dimethylamino- and the triarylamine substitution further improved the reaction outcome (4a vs. $\mathbf{4 b}$ and $\mathbf{4 c}$ vs. $\mathbf{4 b}$ ) and even provided atropisomeric $^{10,6 \mathrm{c}}$ 1-naphthyl substituted acridinium products in high yields $(\mathbf{4 f}, \mathbf{4 j}, \mathbf{4 l})$. We next investigated the photophysical and electrochemical properties of the products. The singlet-excited states of all catalysts possess lifetimes of a few nanoseconds, which is long enough for bimolecular substrate activations. Notably, we observed catalyst tunability in terms of oxidative character in their excited state (Table 2). In agreement with an adjustable $\pi-\pi^{*}$ transition character between the frontier orbitals of the catalysts (as in Figure 1), the novel acridinium dyes $\mathbf{4 a - 1}$ span a wide range of triplet energies $(E T)$ and excited state reduction potentials from $\mathrm{E}_{1 / 2}\left(\mathrm{P}^{*} /\right.$ $\left.\mathrm{P}^{-}\right)=+1.81 \mathrm{~V}(\mathbf{4 b})$ over $\mathrm{E}_{1 / 2}\left(\mathrm{P}^{*} / \mathrm{P}^{-}\right)=+1.40 \mathrm{~V}(\mathbf{4 h}$ and $\mathbf{4 j})$ similar to $\mathrm{Ru}(\mathrm{bpz})_{3}{ }^{2+}$, to $\mathrm{E}_{1 / 2}\left(\mathrm{P}^{*} / \mathrm{P}^{-}\right)=+1.19 \mathrm{~V}$ vs. SCE (4l) with an even lower $\mathrm{E}_{1 / 2}\left(\mathrm{P}^{*} / \mathrm{P}^{-}\right)$than $\operatorname{Ir}\left[\mathrm{dF}\left(\mathrm{CF}_{3}\right) \mathrm{ppy}\right]_{2}(\mathrm{dtbbpy})^{+}$ $\left(\mathrm{E}_{1 / 2}\left(\mathrm{P}^{*} / \mathrm{P}^{-}\right)=+1.21 \mathrm{~V}\right)$.

Table 2: Photophysical and electrochemical properties of the catalysts

\begin{tabular}{ccccccc}
\hline Dye & $\begin{array}{c}E_{0,0^{a}} \\
{[\mathrm{eV}]}\end{array}$ & $\begin{array}{c}E_{\mathrm{T}^{b}} \\
{[\mathrm{eV}]}\end{array}$ & $\begin{array}{c}E_{1 / 2}\left(\mathrm{P} / \mathrm{P}^{-}\right) \\
{[\mathrm{V}]^{c}}\end{array}$ & $\begin{array}{c}E_{1 / 2}\left(\mathrm{P}^{*} / \mathrm{P}^{-}\right) \\
{[\mathrm{V}]^{d}}\end{array}$ & $\begin{array}{c}\tau^{a} \\
{[\mathrm{~ns}]}\end{array}$ & $\begin{array}{c}\text { HOMO-LUMO } \\
\text { transition }{ }^{b}\end{array}$ \\
\hline $\mathbf{1}^{6 \mathbf{a}}$ & 2.40 & 1.89 & -1.15 & +1.25 & 2.2 & $\pi-\pi^{*}$ \\
$\mathbf{4 a}$ & 2.30 & 1.91 & -0.83 & +1.47 & $0.9,4.4$ & $\pi-\pi^{*}$ \\
$\mathbf{4 b}$ & 2.29 & 1.76 & -0.48 & +1.81 & $1.0,3.0,17.3$ & $\pi-\pi^{*}$ \\
$\mathbf{4 c}$ & 2.23 & 1.75 & -0.54 & +1.69 & $1.0,9.9$ & $\pi-\pi^{*}$ \\
$\mathbf{4 d}$ & 2.25 & 1.76 & -0.57 & +1.68 & $1.4,12.1$ & mixed \\
$\mathbf{4 e}$ & 2.25 & 1.75 & -0.56 & +1.69 & $1.2,3.3,16.8$ & mixed \\
$\mathbf{4 f}$ & 2.26 & 1.74 & -0.53 & +1.73 & $1.0,4.5$ & $\mathrm{CT}$ \\
$\mathbf{4 g}$ & 2.29 & 1.90 & -0.89 & +1.40 & $1.0,6.9$ & $\pi-\pi^{*}$ \\
$\mathbf{4 h}$ & 2.29 & 1.91 & -0.89 & +1.40 & $1.1,6.8$ & $\pi-\pi^{*}$ \\
$\mathbf{4 i}$ & 2.29 & 1.91 & -0.90 & +1.39 & $1.1,7.2$ & $\pi-\pi^{*}$ \\
$\mathbf{4 j}$ & 2.27 & 1.88 & -0.87 & +1.40 & $1.0,6.2$ & mixed \\
$\mathbf{4 k}$ & 1.94 & 1.17 & -0.71 & +1.23 & $1.5,5.3$ & $\pi-\pi^{*}$ \\
$\mathbf{4 l}$ & 1.87 & 1.14 & -0.68 & +1.19 & $0.9,5.0$ & $\pi-\pi^{*}$ \\
\hline
\end{tabular}

${ }^{a}$ Measured in $\mathrm{MeCN}\left(15 \mu \mathrm{molL}^{-1}\right) .{ }^{b}$ DFT calculation, experimental ET for 1: $1.91 \mathrm{eV} .{ }^{c}$ Measured in $0.1 \mu \mathrm{molL}^{-1} \mathrm{n}-\mathrm{Bu}_{4} \mathrm{NPF}_{6}$ in degassed, dry MeCN against SCE. ${ }^{d}$ Details are described in the SI. ${ }^{10}$ 
As a rule of thumb, the introduction of $\mathrm{NMe}_{2}$ substituents lowers the $\mathrm{E}_{1 / 2}\left(\mathrm{P}^{*} / \mathrm{P}^{-}\right)$by $0.3-0.4 \mathrm{~V}$ and $\mathrm{OMe}$ groups by $0.1-0.2 \mathrm{~V}$, while differences in substitution patterns show an expected strong influence on the redox behavior.

To test the performance of the modular acridinium catalysts, we comparatively investigated the decarboxylative fluorination of substrates $\mathbf{5 a}$ and $\mathbf{5 b}$ (Scheme 2). ${ }^{16}$ Gratifyingly, the diaminoacridinium catalyst 1 allowed to reduce the reaction time to convert 5a from $60 \mathrm{~h}$ to $6 \mathrm{~h}$ and to halve the catalyst loading to $2.5 \mathrm{~mol} \%$ with respect to previous organocatalytic methods, ${ }^{16 \mathrm{c}}$ approaching the efficiency of optimized polypyridyl Ircatalysts $(1 \mathrm{~mol} \%) .{ }^{16 \mathrm{~b}}$ Interestingly, the complementarity to known acridinium catalysts $(47-52 \%)^{10}$ and donor-acceptor cyanoarene photocatalysts was particularly evident (4CzIPN, $65 \%) .{ }^{17}$ Cognizant of the complex interplay of the numerous catalyst properties impacting their performance, tentative correlations indicate the merits of a library based on an identical core structure. The yield was found to increase from $58 \%$ with catalyst 4e $\left(\mathrm{E}_{1 / 2}\left(\mathrm{P} / \mathrm{P}^{-}\right)=-0.56 \mathrm{~V} ; \mathrm{E}_{1 / 2}\left(\mathrm{P}^{*} / \mathrm{P}^{-}\right)=+1.69 \mathrm{~V}\right), 75 \%$ with 6-aminoacridinium $4 \mathbf{h}\left(\mathrm{E}_{1 / 2}\left(\mathrm{P} / \mathrm{P}^{-}\right)=-0.89 \mathrm{~V} ; \mathrm{E}_{1 / 2}\left(\mathrm{P}^{*} / \mathrm{P}^{-}\right)=\right.$ $+1.40 \mathrm{~V})$ to $80 \%$ with catalyst $1\left(\mathrm{E}_{1 / 2}\left(\mathrm{P} / \mathrm{P}^{-}\right)=-1.15 \mathrm{~V} ; \mathrm{E}_{1 / 2}\right.$ $\left.\left(\mathrm{P}^{*} / \mathrm{P}^{-}\right)=+1.25 \mathrm{~V}\right)$. 7-Aminoacridinium catalyst $4 \mathbf{k}$ with a different substitution pattern eludes this trend, possibly due to the weaker absorption of the catalyst at the peak emission wavelength of the light source. Strikingly, changing to substrate $\mathbf{5 b}$ required acridinium salts $4 \mathbf{e}$ or $4 \mathbf{f}\left(\mathrm{E}_{1 / 2}\left(\mathrm{P} / \mathrm{P}^{-}\right)=-0.56 \mathrm{~V} ;-0.53\right.$ $\left.\mathrm{V} ; \mathrm{E}_{1 / 2}\left(\mathrm{P}^{*} / \mathrm{P}^{-}\right)=+1.69 \mathrm{~V} ;+1.73 \mathrm{~V}\right)$ for optimal reaction conditions (93\% and $94 \%$ yield) akin to the described performance of distinct polypyridyl transition metal catalyst in this reaction, ${ }^{16 a, b}$ further underscoring the importance of modularity in the acridinium catalysts.
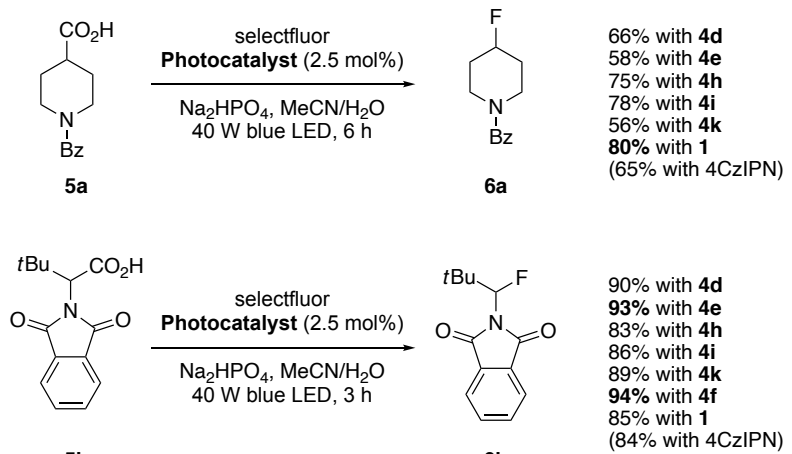

$5 b$

$6 b$

\section{Scheme 2. Comparative acridinium catalyzed decarboxyla-}

tive fluorination. ${ }^{10}$

These findings were substantiated by an unprecedented organophotocatalytic variant of Yoon's [3+2]-cycloaddition induced by the oxidation of phenol 7 to form 2,3-dihydrobenzofuran $( \pm)-9$ (Scheme 3). ${ }^{18}$ The aminoacridinium catalyst $4 \mathrm{~h}$ provided $( \pm)-9$ with a suitably low catalyst loading of $2.5 \mathrm{~mol} \%$, thus representing an efficient organophotocatalytic alternative for this oxidative cycloaddition. ${ }^{10}$ While subtle effects likely also impact the performance of this reaction, the aminomethoxyacridinium catalysts, which are readily accessible by the described synthetic approach (Table 1) and characterized by reduction potentials of around $\left(\mathrm{E}_{1 / 2}\left(\mathrm{P} / \mathrm{P}^{-}\right)=-0.89 \mathrm{~V} ; \mathrm{E}_{1 / 2}\left(\mathrm{P}^{*} / \mathrm{P}^{-}\right)=\right.$ $+1.40 \mathrm{~V})$, appear as optimal catalysts. Highly oxidizing photocatalysts (4d, 4e) might also oxidize the olefinic co-substrate, reducing the overall yield of the transformation.

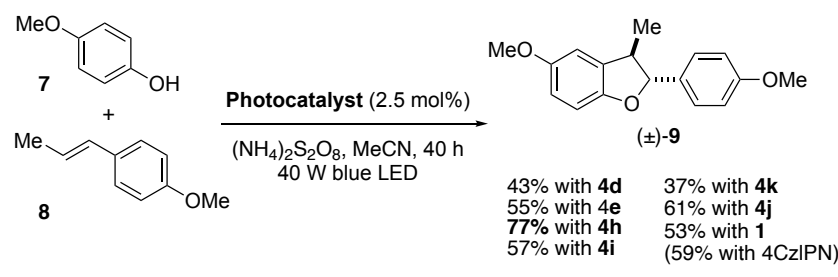

Scheme 3. $[3+2]-C y c l o a d d i t i o n$ catalyzed by an aminoacridinium catalyst. ${ }^{10}$

Consistent with these observations, the decarboxylative dual catalytic cross coupling (Scheme 1) was also observed to be strongly impacted by the substitution pattern of the modular acridinium catalysts (Table 3 ). In this reaction, the examined 6-aminoacridinium catalysts $\mathbf{1}, \mathbf{4 h}$ and $4 \mathbf{i}$ suitably provided C-C cross-coupled product $\mathbf{A}$ with an increased yield when using the milder catalyst $1\left(\mathrm{E}_{1 / 2}\left(\mathrm{P} / \mathrm{P}^{-}\right)=-1.15 \mathrm{~V} ; \mathrm{E}_{1 / 2}\left(\mathrm{P}^{*} / \mathrm{P}^{-}\right)=\right.$ $+1.25 \mathrm{~V})$, whereas the Fukuzumi catalyst, $\mathbf{4 d}$ and $\mathbf{4 e}$ appear as too strongly oxidizing for this multicomponent system.

Table 3: Impact of modularity for acridinium-catalyzed cross couplings ${ }^{a}$

\begin{tabular}{cccccc}
\hline Dye & A & B & Dye & A & B \\
\hline $\mathbf{1}$ & $86 \%$ & - & $\mathbf{4 h}$ & $57 \%$ & $5 \%$ \\
$\mathbf{4 d}$ & - & $3 \%$ & $\mathbf{4 i}$ & $75 \%$ & $6 \%$ \\
$\mathbf{4 e}$ & - & $9 \%$ & $\mathbf{4 k}$ & - & $8 \%$ \\
\hline
\end{tabular}

${ }^{a}$ Dual catalytic cross coupling as shown in Scheme 1.

Notably the differences of $\mathbf{1}$ and the Fukuzumi catalyst observed in the photophysical study were also confirmed in stilbene isomerization reactions using $\mathbf{4 a - 4 1}$ and a dimethylacridinium catalyst, ${ }^{14}$ resulting in significant variation of the isomerization efficiency (17-84\% yield for cis-stilbene, Table 4). ${ }^{10}$ While the distinction between TTET (triplet mechanism) ${ }^{9 \mathrm{f}}$ and electron transfer driven (radical cation mechanism) ${ }^{4 \mathrm{c}}$ stilbene isomerization reactions remains tentative, the most highly oxidizing acridinium catalysts with $\mathrm{CT}$ excited states were found to result in low cis isomer yields $\left(4 \mathrm{e},\left(\mathrm{E}_{1 / 2}\left(\mathrm{P} / \mathrm{P}^{-}\right)=-0.56 \mathrm{~V}\right.\right.$; $\left.\mathrm{E}_{1 / 2}\left(\mathrm{P}^{*} / \mathrm{P}^{-}\right)=+1.69 \mathrm{~V}, 16 \%\right)$. In contrast, low excited-state reduction potentials, $\pi-\pi^{*}$ states and high triplet energies appear as promising catalyst properties for accumulating the thermodynamically less stable cis-stilbene isomer $(\mathbf{4 k}$, $\left(\mathrm{E}_{1 / 2}\left(\mathrm{P} / \mathrm{P}^{-}\right)=-0.71 \mathrm{~V} ; \mathrm{E}_{1 / 2}\left(\mathrm{P}^{*} / \mathrm{P}^{-}\right)=+1.23 \mathrm{~V}, 84 \%\right)$.

Table 4: Acridinium stilbene isomerization

\begin{tabular}{cccccc}
\hline Dye & $\begin{array}{c}\text { cis- } \\
\text { stilbene }\end{array}$ & Dye & $\begin{array}{c}\text { cis- } \\
\text { stilbene }\end{array}$ & Dye & $\begin{array}{c}\text { cis- } \\
\text { stilbene }\end{array}$ \\
\hline $\mathbf{1}$ & $78 \%$ & $\mathbf{4 e}$ & $16 \%$ & $\mathbf{4 j}$ & $54 \%$ \\
$\mathbf{4 a}$ & $47 \%$ & $\mathbf{4 f}$ & $45 \%$ & $\mathbf{4 k}$ & $\mathbf{8 4 \%}$ \\
$\mathbf{4 b}$ & $17 \%$ & $\mathbf{4 g}$ & $41 \%$ & $\mathbf{4 I}$ & $77 \%$ \\
$\mathbf{4 c}$ & $37 \%$ & $\mathbf{4 h}$ & $72 \%$ & $\begin{array}{c}\text { dimethyl- } \\
\text { acridinium }\end{array}$ & $28 \%$ \\
$\mathbf{4 d}$ & $36 \%$ & $\mathbf{4 i}$ & $70 \%$ & $\begin{array}{c}\text { Fukuzumi } \\
\text { catalyst }\end{array}$ & $18 \%$ \\
\hline
\end{tabular}


The combined effects of the numerous catalyst properties, such as excited-state energies, redox potentials, excited-state lifetimes, extinction coefficients, catalyst stabilities and the photoactivities of degradation products, underline the value of tunability within a class of photocatalysts for the design and optimization of photocatalytic reactions. Moreover, photostable catalysts significantly reduce further uncertainties, which prompted us to investigate the inherent photostabilities of the prepared acridinium dyes. Good to excellent intrinsic catalyst stabilities that compare favorably to $\mathrm{Ru}(\mathrm{bpy})_{3}{ }^{2+}$ were observed in the photostability assay (see SI page S38 for details).

\section{CONCLUSIONS}

In conclusion, divergent organophotocatalytic reaction pathways investigated in detail by photophysical studies revealed the hallmarks of aminoacridinium catalysts and the contributions of singlet and triplet excited state kinetics. Moreover, the modularity of organophotocatalysts to complement tunable polypyridyl transition metal complexes was emphasized by a versatile acridinium synthesis using bifunctional organodilithium reagents from the combination of X-M exchange and directed ortho-metalation. Twelve novel acridinium catalysts with charge transfer or $\pi-\pi^{*}$ lowest-energetic electronic transitions, high inherent photostabilities, adjustable triplet energies and a broad range of excited state reduction potentials were prepared. The virtues of modular organophotocatalysts were evident by optimal catalytic features to refine photoredox methodology. With the growing scope of complementary families of organophotocatalysts, significant contributions to reaction discovery, sustainable synthesis and scale-up can be anticipated. Our current studies focus on the applications of the novel organophotocatalysts and the design of particularly photostable aminoacridinium catalysts.

\section{AUTHOR INFORMATION}

\section{Corresponding Authors}

* Email: oliver.wenger@unibas.ch, christof.sparr@unibas.ch

\section{Author Contributions}

The manuscript was written through contributions of all authors. All authors have given approval to the final version of the manuscript. \$These authors contributed equally.

\section{Funding Sources}

Swiss National Science Foundation (BSSGI0-155902/1 and 175746), German National Academy of Sciences Leopoldina, NCCR Molecular Systems Engineering

\section{Notes}

The authors declare no conflict of interest. Compounds 4a-l are part of a filed patent (C. Fischer, C. Sparr. EP 17/188,288) licensed to Solvias. The catalysts will be commercially available.

\section{ASSOCIATED CONTENT}

\section{Supporting Information}

The Supporting Information is available free of charge on the ACS Publications website.

Experimental procedures, compound characterization, divergent pathways, DFT calculations, photophysical properties, quenching studies, emission lifetime determinations, benchmarking reactions and inherent photostability studies (PDF)

\section{ACKNOWLEDGMENTS}

We gratefully acknowledge the Swiss National Science Foundation (BSSGI0-155902/1 and 175746), the University of Basel, the German National Academy of Sciences Leopoldina and the NCCR Molecular Systems Engineering for financial support. We thank Prof. M. Mayor for equipment usage and Thomas Buchholz and Dragan Miladinov for skillful experimental support.

\section{REFERENCES}

(1) (a) Prier, C. K.; Rankic, D. A.; MacMillan, D. W. C. Visible Light Photoredox Catalysis with Transition Metal Complexes: Applications in Organic Synthesis. Chem. Rev. 2013, 113, 5322-5363. (b) Narayanam, M. R.; Stephenson, C. R. J. Visible Light Photoredox Catalysis: Applications in Organic Synthesis. Chem. Soc. Rev. 2011, 40, 102-113. (c) Barton, D. H. R.; Csiba, M. A.; Jaszberenyi J. Cs. $\mathrm{Ru}$ (bpy) $3^{2+}$-mediated Addition of Se-phenyl $p$-Tolueneselenosulfonate to Electron Rich Olefins. Tetrahedron Lett. 1994, 35, 2869-2872. (d) Ma, J.; Zhang, X.; Huang, X.; Luo, S.; Meggers, E. Preparation of Chiral-at-metal Catalysts and their use in Asymmetric Photoredox Chemistry. Nat. Protoc. 2018, 13, 605-632. (e) Milligan, J. A.; Phelan, J. P.; Badir, S. O.; Molander G. Alkyl Carbon-Carbon Bond Formation by Nicke1/Photoredox Cross-Coupling. Angew. Chem. Int. Ed. 2019, 58, 6152-6163. (f) Reckenthäler, M.; Griesbeck, A. G. Photoredox Catalysis for Organic Syntheses. Adv. Synth. Catal. 2013, $355,2727-2740$.

(2) (a) Arias-Rotondo, D. M.; McCusker, J. K. The Photophysics of Photoredox Catalysis: A Roadmap for Catalyst Design. Chem. Soc. Rev. 2016, 45, 5803-5820. (b) Buzzetti, L.; Crisenza, G. E. M.; Melchiorre, P. Mechanistic Studies in Photocatalysis. Angew. Chem. Int. Ed. 2019, 58, 3730-3747.

(3) (a) Marzo, L.; Pagire, S. K.; Reiser, O.; König, B. Visible-Light Photocatalysis: Does It Make a Difference in Organic Synthesis? Angew. Chem. Int. Ed. 2018, 57, 10034-10072. (b) Brasholz, M. Acridinium Dyes and Quinones in Photocatalysis. In Science of Synthesis: Photocatalysis in Organic Synthesis, (Ed.: König, B.), Thieme, Stuttgart, 2019, pp. 371-389. (c) Metternich, J. B.; Mudd, R. J.; Gilmour R. Flavins in Photochemistry. In Science of Synthesis: Photocatalysis in Organic Synthesis, (Ed.: König, B.), Thieme, Stuttgart, 2019, pp. 391-404. (d) Metternich, J. B.; Artiukhin, D. G.; Holland, M. C.; von Bremen-Kühne, M.; Neugebauer, J.; Gilmour, R. Photocatalytic $\mathrm{E} \rightarrow \mathrm{Z}$ Isomerization of Polarized Alkenes Inspired by the Visual Cycle: Mechanistic Dichotomy and Origin of Selectivity. $J$. Org. Chem. 2017, 82, 9955-9977. (e) Hari, D. P.; König, B. Synthetic Applications of Eosin Y in Photoredox Catalysis. Chem. Commun. 2014, 50, 6688-6699.

(4) (a) Fukuzumi, S.; Ohkubo, K.; Suenobu, T.; Kato, K.; Jujitsuka, M.; Ito, O. Photoalkylation of 10-Alkylacridinium Ion via a ChargeShift Type of Photoinduced Electron Transfer Controlled by Solvent Polarity. J. Am. Chem. Soc. 2001, 123, 8459-8467. (b) Fukuzumi, S.; Kotani, H.; Ohkubo, K.; Ogo, S.; Tkachenko, N. V.; Lemmetyinen, H. Electron-Transfer State of 9-Mesityl-10-methylacridinium Ion with a Much Longer Lifetime and Higher Energy Than That of the Natural Photosynthetic Reaction Center. J. Am. Chem. Soc. 2004, 126, 16001601. (c) Fukuzumi, S.; Ohkubo, K. Organic Synthetic Transformations Using Organic Dyes as Photoredox Catalysts. Org. Biomol. Chem. 2014, 12, 6059-6071.

(5) (a) Romero, N. A.; Nicewicz, D. A. Organic Photoredox Catalysis. Chem. Rev. 2016, 116, 10075-10166. (b) Joshi-Pangu, A.; Lévesque, F.; Roth, H. G.; Oliver, S. F.; Campeau, L.-C.; Nicewicz, D. A.; DiRocco, D. A. Acridinium-Based Photocatalysts: A Sustainable Option in Photoredox Catalysis. J. Org. Chem. 2016, 81, 7244-7249. (c) Gini, A.; Uygur, M.; Rigotti, T.; Alemán, J.; García Mancheño, O. Novel Oxidative Ugi Reaction for the Synthesis of Highly Active, Visible-Light, Imide-Acridinium Organophotocatalysts. Chem. Eur. J. 2018, 24, 12509-12514. (d) Uraguchi, D.; Torii, M.; Ooi, T. Acridinium Betaine as a Single-Electron-Transfer Catalyst: Design and Application to Dimerization of Oxindoles. ACS Catal. 2017, 74, 2765-2769. (e) White, A. R.; Wang, L.; Nicewicz, D. A. Synthesis and Character- 
ization of Acridinium Dyes for Photoredox Catalysis. Synlett 2019, $30,827-832$.

(6) (a) Fischer, C.; Sparr, C. Direct Transformation of Esters into Heterocyclic Fluorophores. Angew. Chem. Int. Ed. 2018, 57, 24362440. (b) Fischer, C.; Sparr, C. Synthesis of 1,5-Bifunctional Organolithium Reagents by a Double Directed ortho-Metalation: Direct Transformation of Esters into 1,8-Dimethoxyacridinium Salts. Tetrahedron 2018, 74, 5486-5493; (c) Fischer, C.; Sparr, C. Configurationally Stable Atropisomeric Acridinium Fluorophores. Synlett 2018, 29, 2176-2180.

(7) Zuo, Z.; Ahneman, D. T.; Chu, L.; Terrett, J. A.; Doyle, A. G.; MacMillan, D. W. C. Merging Photoredox with Nickel Catalysis: Coupling of $\alpha$-Carboxyl $\mathrm{sp}^{3}$-Carbons with Aryl Halides. Science 2014, $345,437-440$.

(8) (a) McCarthy, B. G.; Pearson, R. M.; Lim, C.-H.; Sartor, S. M.; Damrauer, N. H.; Miyake, G. M. Structure-Property Relationships for Tailoring Phenoxazines as Reducing Photoredox Catalysts. J. Am. Chem. Soc. 2018, 140, 5088-5101. (b) Speckmeier, E.; Fischer, T. G.; Zeitler, K. A Toolbox Approach To Construct Broadly Applicable Metal-Free Catalysts for Photoredox Chemistry: Deliberate Tuning of Redox Potentials and Importance of Halogens in Donor-Acceptor Cyanoarenes. J. Am. Chem. Soc. 2018, 140, 15353-15365.

(9) (a) Welin, E. R.; Le, C. C.; Arias-Rotondo, D. M.; McCusker, J. K.; MacMillan, D. W. C. Photosensitized, Energy Transfer-mediated Organometallic Catalysis through Electronically Excited Nickel(II). Science 2017, 355, 380-385. (b) Kim, T.; McCarver, S. J.; Lee, C.; MacMillan, D. W. C. Sulfonamidation of Aryl and Heteroaryl Halides through Photosensitized Nickel Catalysis. Angew. Chem. Int. Ed. 2018, 57, 3488-3492. (c) Yoo, W.-J.; Tsukamoto, T.; Kobayashi, S. Visible Light-Mediated Ullmann-Type C-N Coupling Reactions of Carbazole Derivatives and Aryl Iodides. Org. Lett. 2015, 17, 36403642. (d) Heitz, D. R.; Tellis, J. C.; Molander, G. A. Photochemical Nickel-Catalyzed C-H Arylation: Synthetic Scope and Mechanistic Investigations. J. Am. Chem. Soc. 2016, 138, 12715-12718. (e) Sun, Z.-D.; Kumagai, N.; Shibasaki, M. Photocatalytic $\alpha$-Acylation of Ethers. Org. Lett. 2017, 19, 3727 - 3730. (f) Strieth-Kalthoff, F.; James, M. J.; Teders, M.; Pitzer, L.; Glorius, F. Energy Transfer Catalysis Mediated by Visible Light: Principles, Applications, Directions. Chem. Soc. Rev. 2018, 47, 7190-7202.

(10) See Supporting Information for details

(11) Luo, J.; Zhang, J. Donor-Acceptor Fluorophores for VisibleLight-Promoted Organic Synthesis: Photoredox/Ni Dual Catalytic $\mathrm{C}\left(\mathrm{sp}^{3}\right)-\mathrm{C}\left(\mathrm{sp}^{2}\right)$ Cross-Coupling. ACS Catal. 2016, 6, 873-877.

(12) Kerzig, C.; Wenger, O. S. Sensitized Triplet-Triplet Annihilation Upconversion in Water and its Application to Photochemical Transformations. Chem. Sci. 2018, 9, 6670-6678.

(13) Penzkofer, A.; Beidoun, A.; Daiber, M. Intersystem-Crossing and Excited-State Absorption in Eosin Y Solutions Determined by Picosecond Double Pulse Transient Absorption Measurements. J. Lumin. 1992, 51, 297-314.

(14) (a) Romero, N. A.; Nicewicz, D. A. Mechanistic Insight into the Photoredox Catalysis of Anti-Markovnikov Alkene Hydrofunctionalization Reactions. J. Am. Chem. Soc. 2014, 136, 17024-17035. (b) Benniston, A. C.; Harriman, A.; Li, P.; Rostoron, J. P.; van Ramesdonk, H. J.; Groeneveld, M. M.; Zhang, H.; Verhoeven, J. W. Charge Shift and Triplet State Formation in the 9-Mesityl-10methylacridinium Cation. J. Am. Chem. Soc. 2005, 127, 1605416064.

(15) (a) Saltiel, J.; Hammond, G. S. Mechanisms of Photochemical Reactions in Solution. XVII. cis-trans Isomerization of the Stilbenes by Excitation Transfer from Low Energy Sensitizers. J. Am. Chem. Soc. 1963, 85, 2515-2516. (b) Montalti, M.; Credi, A.; Prodi, L.; Gandolfi, M. T. Handbook of Photochemistry, CRC/Taylor \& Francis, Boca Raton, 2006.

(16) (a) Rueda-Becerril, M.; Mahé, O.; Drouin, M.; Majewski, M. B.; West, J. G.; Wolf, M. O.; Sammis, G. M.; Paquin, J.-F. Direct C-F Bond Formation Using Photoredox Catalysis. J. Am. Chem. Soc. 2014, 136, 2637-2641. (b) Ventre, S.; Petronijevic, F. R.; MacMillan, D. W. C. Decarboxylative Fluorination of Aliphatic Carboxylic Acids via Photoredox Catalysis. J. Am. Chem. Soc. 2015, 137, 5654-5657. (c) Wu, X.; Meng, C.; Yuan, X.; Jia, X.; Qian, X.; Ye, J. Transition- metal-free Visible-light Photoredox Catalysis at Room-temperature for Decarboxylative Fluorination of Aliphatic Carboxylic Acids by Organic Dyes. Chem. Commun, 2015, 51, 11864-11867.

(17) Uoyama, H.; Goushi, K.; Shizu, K.; Nomura, H.; Adachi, C. Highly Efficient Organic Light-Emitting Diodes from Delayed Fluorescence. Nature 2012, 492, 234-238.

(18) Blum, T. R.; Zhu, Y.; Nordeen, S. A.; Yoon, T. P. Photocatalytic Synthesis of Dihydrobenzofurans by Oxidative [3+2] Cycloaddition of Phenols. Angew. Chem. Int. Ed. 2014, 53, 11056-11059. 